\title{
Transaction
}

\section{Fabrication of Cellulose Nanofibers from Parenchyma Cells of Pears and Apples}

\author{
Shinsuke Ifuku ${ }^{* 1}$, Masatoshi Adachi ${ }^{* 1}$, Minoru Morimoto ${ }^{* 2}$, and Hiroyuki Saimoto ${ }^{* 1}$ \\ ${ }^{* 1}$ Department of Chemistry and Biotechnology, Graduate School of Engineering, Tottori University, \\ 4-101 Koyama-cho Minami, Tottori 680-8550, Japan \\ ${ }^{* 2}$ Research Center for Bioscience and Technology, Tottori University, \\ 4-101 Koyama-cho Minami, Tottori 680-8550, Japan
}

\begin{abstract}
Cellulose nanofibers were isolated from parenchyma cells of pears and apples by one-time fibrillation using a grinding apparatus after the removal of matrix substances. The fibrillated cellulose samples were observed by field emission scanning electron microscopy, and were found to have a fine nano-structure. That is, the structure was uniform with an average width of approximately $18 \mathrm{~nm}$ and a high aspect ratio. These appearances were similar to those for nanofibers from wood. The fabricated nanofibers were characterized by FT-IR spectra and X-ray diffraction profiles, which showed that cellulose nanofibers were extracted, and the cellulose I crystal structure was maintained after a series of chemical treatments and grinding processing.
\end{abstract}

(Received 22 November, 2010 ; Accepted 19 January, 2011)

\section{Introduction}

Dietary fibers are present in the primary cell walls of edible plants such as fruits and vegetables. Dietary fibers consist of mainly cellulose, hemicellulose, lignin, and pectin. They can be available as functional food ingredients such as swelling and thickening agents, stabilizers, film-forming agents, and health ingredients [1], [2]. Thus, dietary fibers improve the nutritional and the technological properties of foods, and the incorporation of such health ingredients in foods is popular way to prepare functional foods.

Recently, Abe et al. reported an efficient method for isolating cellulose nanofibers with a uniform width of approximately $15 \mathrm{~nm}$ from wood [3]. Because cellulose nanofibers in the cell walls of wood are embedded in matrix substances such as lignin and hemicellulose, the cellulose can be isolated by removal of the matrix substances as nanofibers after a very simple mechanical treatment.

As the authors have described that the nanofiber preparation method can be used to isolate cellulose nanofibers from any natural plant containing lignin and hemicellulose such as flax, sugarcane bagasse, and wheat straw [4],[5], we consider that this isolation method would be universally applicable to any natural nanofiber source consisting of nanofibers and other embedding matrixes. In fact, we have recently succeeded in isolating chitin nanofibers from crab and shrimp shells, since chitin nanofibers are also encased in matrix components such as proteins and minerals [6],[7]. Similarly, the parenchyma cells of fruits and vegetables consist of cellulose nanofiber networks, which are embedded in matrix substances such as hemicellulose polysaccharides, the pectic matrix, and phenolic polymer lignin in the primary cell wall [8]. These matrices contribute to both the physical integrity and physiological status of cell walls. Although we consider that the isolation procedure is applicable to fruits bodies, higher-order structure and role of parenchyma cell wall are quite different from that of wood cell wall. Most important role of parenchyma cell is a store of nutrient.

In the present study, we studied the isolation of natural cellulose nanofibers with a uniform width from parenchyma cells of pears and apples. It is known that pear and apple have relatively abundant dietary fibers (2 3 wt\% with wet weight basis) [9]. Nanometer-sized dietary fibers from edible plants with a very high surfaceto-volume ratio will be developed into novel functional food ingredients.

\section{Materials and Methods}

\subsection{Materials}

Mature Nijuseiki pear, Shinju pear, and Ourin apple were obtained from a local supermarket. All chemicals were purchased from Aldrich or Kanto chemical and used without further purification. 


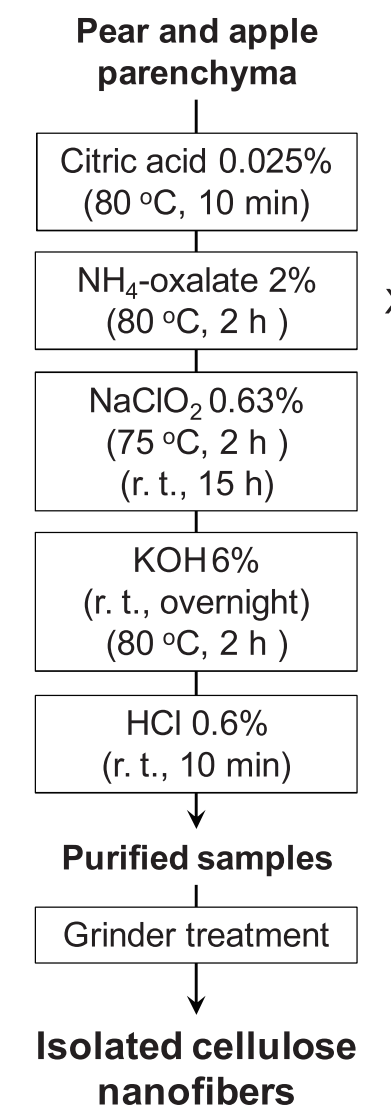

Fig. 1 Preparation procedure of cellulose nanofibers from pear and apple.

\subsection{Preparation of cellulose nanofibers}

Pear and apple were purified to isolate the cellulose component according to the general method [10]. The samples were purified by a series of chemical treatments according to the flowchart shown in Fig. 1. In general, the drying process of cellulose fibers causes strong hydrogen bonding between these fibers after a removal of matrix, which makes it difficult to fibrillate them to nanofibers. Therefore, the sample must be kept wet after removal of the matrix for nanofiber preparation. The peeled pear and apple parenchyma was first roughly crushed with a domestic blender, and was filtered and washed thoroughly with distilled water to remove the watersoluble ingredients. The mash was blanched in $0.025 \%$ $(\mathrm{w} / \mathrm{v})$ citric acid for $10 \mathrm{~min}$. at $80{ }^{\circ} \mathrm{C}$. After filtration and rinsing with distilled water, the residue was treated in $2 \%$ $(\mathrm{w} / \mathrm{v})$ ammonium oxalate ( $\mathrm{pH} 5.0$ ) for 2 hours at $80{ }^{\circ} \mathrm{C}$ to remove the pectin matrix. After the second extraction with ammonium oxalate, the residue was mixed with water, $0.63 \%(\mathrm{w} / \mathrm{v})$ sodium chlorite, and $0.3 \%(\mathrm{v} / \mathrm{v})$ acetic acid. The mixture was stirred at $75^{\circ} \mathrm{C}$ for $2 \mathrm{~h}$ and then washed with distilled water. The extraction was repeated at room temperature for $15 \mathrm{~h}$, and the residue was washed with distilled water. The sample, extracted with chelating, oxidizing, and alkaline agents, was treated with $6 \%(\mathrm{w} / \mathrm{v})$
$\mathrm{KOH}$ at room temperature overnight, filtered, and then extracted again for 2 hours at $80{ }^{\circ} \mathrm{C}$. The residue was then treated with $0.6 \%(\mathrm{w} / \mathrm{w}) \mathrm{HCl}$ at room temperature for $10 \mathrm{~min}$ and washed with distilled water. After the series of chemical treatments, the samples were filtered and rinsed with distilled water until the residues were neutralized.

The purified slurry with $1 \%(\mathrm{w} / \mathrm{v})$ was passed through a grinder (MKCA6-3; Masuko Sangyo Co., Ltd.) at $1500 \mathrm{rpm}$. Grinder treatment was performed with a clearance gauge of -1.5 (corresponding to a $0.15 \mathrm{~mm}$ shift) from the zero position. The position was determined as the point of slight contact between the two grinding stones.

\subsection{Fourier transform infrared (FT-IR) spectroscopy}

Infrared spectra of the cellulose samples were recorded using potassium bromide pellets with an FT-IR spectrometer (FTIR 8300, Shimadzu). All the spectra were obtained by an accumulation of 20 scans, with a resolution of $2 \mathrm{~cm}^{-1}$ at $400-4000 \mathrm{~cm}^{-1}$.

\section{$2.4 \mathrm{X}$-ray diffraction}

$\mathrm{X}$-ray diffraction profiles of the cellulose nanofibers were obtained with $\mathrm{Ni}$-filtered $\mathrm{Cu} \mathrm{K} \alpha$ from an X-ray generator (Shimadzu XRD-6000) operating at $40 \mathrm{kV}$ and $30 \mathrm{~mA}$. The diffraction profile was detected using an Xray goniometer scanning from $5^{\circ}$ to $40^{\circ}$.

\subsection{Scanning electron microscopy (SEM)}

Prepared cellulose nanofiber slurries were diluted with the EtOH and dried in the oven. The cast film was coated with an approximately $2 \mathrm{~nm}$ layer of platinum by an ion sputter coater and observed with a field emission scanning electron microscope (JSM-6700F ; JEOL, Ltd) operating at $1.5 \mathrm{kV}$. The average diameter of isolated nanofibers was estimated from the diameters of 30 nanofibers obtained by manual measurement.

\section{Results and discussion}

\subsection{Fabrication of cellulose into nanofibers}

Nijuseiki pears, which are an indigenous product in Tottori prefecture, were purified by a series of chemical treatments to remove the matrix substances according to the method of Vetter \& Kunzek [10]. Ammonium oxalate was used as the chelating agent to solubilize pectic substances. The extraction step with $\mathrm{NaClO}_{2}$ and acetic acid can remove polyphenolic substances and lignin. The treatment with $\mathrm{KOH}$ can solubilize hemicelluloses and further part of the pectic substances. It is known that almost matrix substances can be removed, and that 

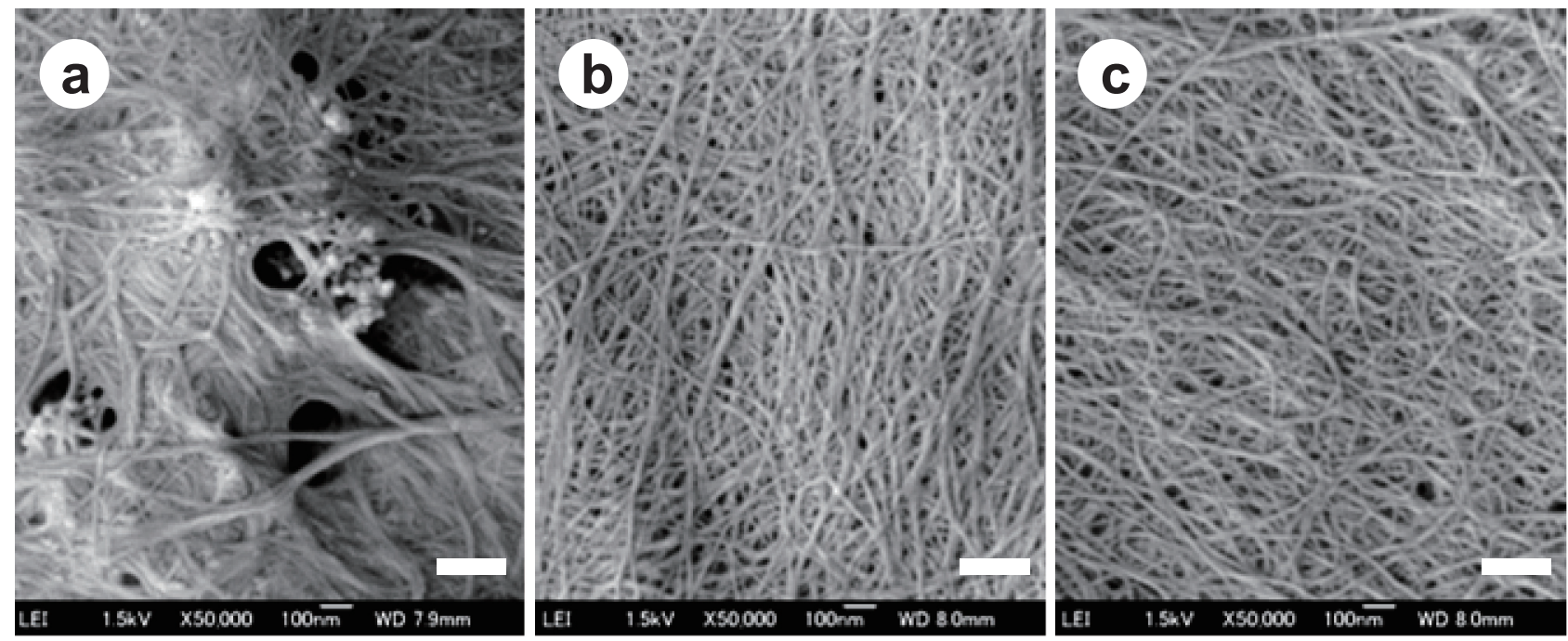

Fig. 2 FE-SEM micrographs of (a) Nijuseiki pear mash before removing matrix components, and purified cellulose fibers (b) before and (c) after one pass through the grinder. The length of scale bars are $200 \mathrm{~nm}$.

cellulose is isolated by this process [10]. Figure 2 shows FE-SEM images of pear mash (a) before and (b) after removing matrix components without grinding treatment. The samples were dried in an oven for SEM observation after solvent replacement from water to EtOH to prevent coagulation between fibers. In Fig. 2a, it can be observed that cellulose microfibrils are embedded in matrix substances in the cell-wall of the pear pulp. The matrix substances such as hemicellulose and pectin behave as adhesives of the microfibrils to make a tough and flexible cell wall. On the other hand, after a series of chemical treatments, a nanofiber network with many air voids was observed after the removal of matrix substances (Fig. 2b). We can see that the layers of linear nanofibers oriented overlap across each other. The widths of the fibers were widely distributed over a range from 10 to $50 \mathrm{~nm}$, which was larger than those of cellulose nanofibers isolated from wood [3]. The thicker fibers are thought to be due to the aggregation of cellulose nanofibers.

The purified cellulose was passed through a grinder with a concentration of $1 \mathrm{wt} \%$ to fibrillate the cellulose fiber network. Interestingly, although cellulose is known as the insoluble component of dietary fiber, the cellulose slurry thus obtained was homogeneously and stably dispersed in water, and had high viscosity similar to the cellulose nanofibers prepared from wood. It appears that fibrillation was accomplished based on the high dispersion properties of the nanofibers in water and their high surface-to-volume ratio. Figure $2 \mathrm{c}$ shows a SEM image of purified cellulose fibers after one pass through the grinder. The isolated cellulose is observed as highly uniform nanofibers with an average width of $18.5 \mathrm{~nm}$, including a $2 \mathrm{~nm}$ platinum coating layer. The appearance of the fibers was very similar to that of cellulose nanofibers prepared from wood [3]. The thicker fibers observed in Fig. $2 b$ were effectively fibrillated by grinder treatment, and the fibers larger than approximately $25 \mathrm{~nm}$ in width were not found. The regularly and linearly oriented fiber network was randomized nonlinearly by fibrillation. Recently, Kuga et al. isolated 1.0-2.0 nm cellulose fibers from several fruit tissues, which is much thinner than our result [12]. Although the size difference may be attributed to the difference of species of fruits or difference of manufacturing equipment for nanofiber production, the most plausible explanation is difference of observation methods. Kuga et al. chose atomic force microscopy (AFM) for nanofiber observation. Since width of single fiber could be directly determined from the contour plot without platinum coating, AFM could estimate the fiber width to be lower than FE-SEM.

The preparation method for cellulose nanofibers was found to be applicable to other fruit bodies. Shinju pear and Ourin apple were purified by removal of the matrix substances according to the same procedure. Figure 3a and $3 \mathrm{~b}$ show SEM images of Shinju pear and Ourin apple after removing matrix components before grinder treatment, respectively. It can be observed that the primary cell-walls were made up of a regularly structured cellulose nanofiber network similar to that of the Nijuseiki pear. Figure 3c and 3d show SEM images of cellulose fibers from Shinju pear and Ourin apple, respectively after one pass through the grinder. The regularly oriented fiber network was completely collapsed, indicating that fine cellulose nanofibers were successfully isolated from Shinju pear and Ourin apple. The average widths of the nanofibers from these samples were $17.1 \mathrm{~nm}$ and $18.8 \mathrm{~nm}$, respectively, which were similar to the nanofibers from Nijuseiki pear. Thus, the grinder 


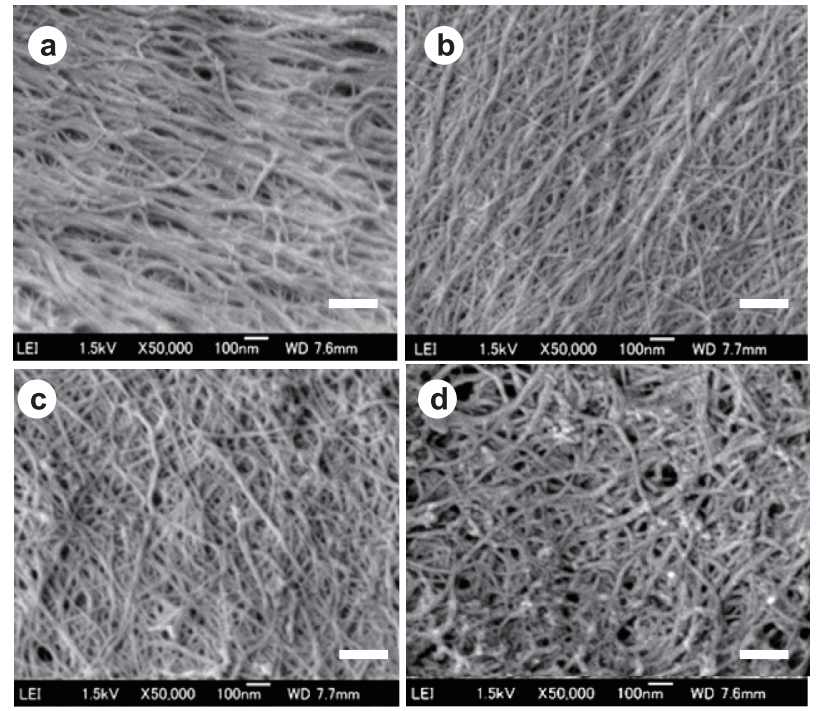

Fig. 3 FE-SEM micrographs of (a) Shinju pear and (b) Ourin apple after removing matrix components, and isolated cellulose fibers of (c) Shinju pear and (d) Ourin apple after one pass through the grinder. The scale bars are $200 \mathrm{~nm}$ in length.

treatment fibrillated the primary cell-wall structures after removal of the matrix substances, enabling us to isolate the individualized cellulose nanofibers from the parenchyma cells of fruit bodies.

\subsection{Characterization of cellulose nanofibers.}

Figure 4 shows the FT-IR spectra of cellulose nanofibers prepared from (a) Nijuseiki pear, (b) Shinju pear, (c) Ourin apple, and (d) commercially available cellulose. The spectra of prepared cellulose nanofibers are in excellent agreement with the spectrum of commercial pure cellulose. An O-H stretching band in the range from 3000 to $3650 \mathrm{~cm}^{-1}$, a C-H stretching in methyl and methylene groups at $2900 \mathrm{~cm}^{-1}, \mathrm{C}-\mathrm{H}$ deformation at $1427 \mathrm{~cm}^{-1}$, O-H in-plane deformation at $1337 \mathrm{~cm}^{-1}, \mathrm{CH}_{2}$ wagging at $1317 \mathrm{~cm}^{-1}$, glucose ring stretching at $1113 \mathrm{~cm}^{-1}, \mathrm{C}-\mathrm{O}$ stretching at $1059 \mathrm{~cm}^{-1}, \mathrm{C}-\mathrm{O}$ stretching at $1033 \mathrm{~cm}^{-1}$, glucose ring stretching and $\mathrm{C}_{1}-\mathrm{H}$ deformation band at $897 \mathrm{~cm}^{-1}$ of the cellulose nanofibers are observed [11]. These absorption peaks are characteristic of cellulose molecules. However, absorption peak observed at around $1650 \mathrm{~cm}^{-1}$ corresponding to $\mathrm{C}=\mathrm{O}$ stretching vibration mode indicates that small portion of hemicellulose or pectin still remains in cellulose even after a series of treatments [12].

Figure 5 shows the diffraction profiles of the cellulose nanofibers prepared from (a) Nijuseiki pear, (b) Shiju pear, (c) Ourin apple, and (d) commercially available pure cellulose. The crystallinity of prepared cellulose nanofibers appeared to be lower than that of commercial cellulose, as the amorphous part of

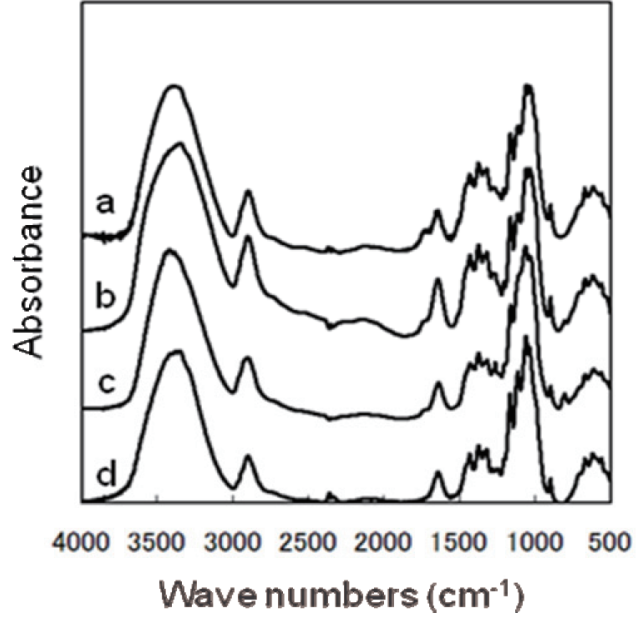

Fig. 4 FT-IR spectra of cellulose nanofibers prepared from (a) Nijuseiki pear, (b) Shinju pear, (c) Ourin apple, and (d) commercially available cellulose.

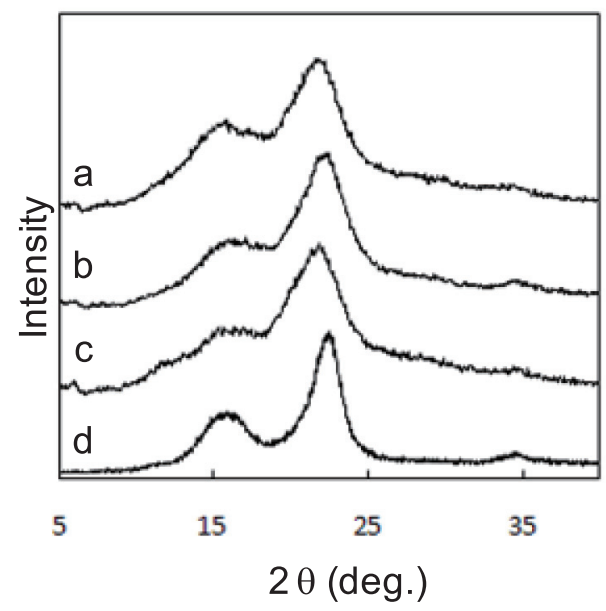

Fig. 5 X-ray diffraction profiles of cellulose nanofibers prepared from (a) Nijuseiki pear, (b) Shinju pear, (c) Ourin apple, and (d) commercially available cellulose.

commercial pure cellulose is almost entirely removed by acid hydrolysis through the purification process. However, the diffraction peaks of cellulose nanofibers observed from $15^{\circ}$ to $17^{\circ}$, and at $22^{\circ}$, which correspond to $110,1-10$, and 200 planes, respectively, are typical crystal patterns of Cellulose I and are coincident with commercial cellulose [13]. Thus, cellulose nanofibers were extracted from the pears and apples, and Cellulose I crystalline structures were maintained after both removal of the matrix and the grinder treatment.

\section{Conclusions}

In this study, cellulose nanofibers were isolated from parenchyma cells of pears and apples. FE-SEM 
observations revealed that the cellulose nanofibers from these samples have uniform structures with a width of approximately $18 \mathrm{~nm}$ and a high aspect ratio, the same as nanofibers prepared from wood. These findings indicate that grinding treatment after matrix removal is applicable to several fruit bodies to isolate cellulose nanofibers. The simple preparation method allows us to obtain homogeneous cellulose nanofibers in parenchyma cells from edible plants. Because these nanofibers, which are so-called nano-sized dietary fibers, have a very high surface-to-volume ratio, high viscosity, and high dispersibility in water, we expect that they will be available as functional food ingredients and will improve the nutritional and the technological properties of foods. And this will promote effective use of unmarketable imperfect produce.

\section{Acknowledgement}

This work was financially supported by KAKENHI (20559003) of JSPS.

\section{References}

1. H. Kunzek, S. Müller, S. Vetter, and R. Godeck, Eur. Food Res. Technol., 214, 361 (2002).

2. F. Guillon, and M. Champ, Food Res. Int., 33, 233. (2000).

3. K. Abe, H. Iwamoto, and H. Yano, 8, 3276, (2007).

4. K. Abe, and H. Yano, Cellulose, 16, 1017 (2009).

5. K. Abe, and H. Yano, Cellulose, 17, 271 (2010).

6. S. Ifuku, M. Nogi, K. Abe, M. Yoshioka, M. Morimoto, H. Saimoto, and H. Yano, Biomacromol., 10, 1584 (2009).

7. S. Ifuku, M. Nogi, K. Abe, M. Yoshioka, M. Morimoto, H. Yano, and H. Saimoto, Carbohydr. Polym., 81, 134 (2010).

8. M. C. McCann, B. Eells, and K. Roberts, J. Cell Sci., 96, 323 (1990).

9. S. Ito, "Science of Fruits" . Asakura publishing. pp 103-108 (1991).

10. S. Vetter, and H. Kunzek, Eur. Res. Technol., 217, 392 (2003).

11. K. K. Pandey, J. Appl. Polym. Sci., 71, 1969 (1999).

12. H. Niimura, T. Yokoyama, S. Kimura, Y. Matsumoto, and S. Kuga, Cellulose, 17, 13 (2010).

13. A. Isogai, "Material Science of Cellulose". University of Tokyo Press, p5-13 (2001). 